\title{
The effects of inhaled NO on plasma vasoactive factor and CTnI level in rabbits with acute massive pulmonary embolism ${ }^{1}$
}

\author{
Zeming Zhang', Zheng Li", Lu Chen"', Yancun Wang'
}

'PhD, Department of Respiratory Medicine, Affiliated Zhou Pu Hospital, Shanghai Health Medical College, China. Conception of the study, technical procedures, manuscript writing, critical revision.

"Master, Department of Respiratory Medicine, Affiliated Hospital, Hebei University, Baoding, China. Technical procedures, analysis of data, manuscript writing.

"'Bachelor, Department of Respiratory Medicine, Affiliated Hospital, Hebei University, Baoding, China. Technical procedures, chart making.

IV Bachelor's degree, Department of Neurology, Affiliated Zhou Pu Hospital, Shanghai Health Medical College, China. Acquisition and interpretation of data.

\section{Abstract}

Purpose: To investigate changes in the plasma concentrations of cardiac troponin I (CTnl), thromboxane A2 (TXA2), prostaglandin 12 (PGI2) and endothelin-1 (ET-1) in rabbits with massive pulmonary embolism (AMPE) and the impact of nitric oxide inhalation (NOI) on these indices.

Methods: A total of 30 Japanese rabbits were used to construct an MPE model and were divided into 3 groups equally ( $n=10)$, including an EXP group (undergoing modeling alone), an NOI group (receiving NOI $2 \mathrm{~h}$ post-modeling) and a CON group (receiving intravenous physiological saline).

Results: In the model group, plasma concentration of CTnl peaked at $16 \mathrm{~h}$ following modeling $(0.46 \pm 0.10 \mu \mathrm{g} / \mathrm{ml})$ and significantly decreased following NOI. Plasma levels of TXB2, PGI2 and ET-1 peaked at 12,16 and $8 \mathrm{~h}$ following modeling, respectively, and significantly decreased at different time points $(0,2,4,8,12,16,20$ and $24 \mathrm{~h})$ following NOI. A significant correlation was observed between the peak plasma CTnl concentration and peak TXB2, 6-keto prostaglandin $\mathrm{F} 1 \alpha$ and $\mathrm{ET}-1$ concentrations in the model and NOI groups.

Conclusion: Increases in plasma TXA2, PGI2 and ET-1 levels causes myocardial damage in a rabbit model of $\mathrm{AMPE}$; however, $\mathrm{NOI}$ effectively down regulates the plasma concentration of these molecules to produce a myocardial-protective effect.

Key words: Pulmonary Embolism. Nitric Oxide. Thromboxane A2. Epoprostenol. Endothelin-1. Rabbits. 


\section{- Introduction}

Acute massive pulmonary embolism (AMPE) is associated with high early mortality ${ }^{1,2}$ of $~ 30 \%$, particularly in patients who present with concomitant shock and heart failure ${ }^{3,4}$. Therefore, early assessment of mortality risk as well as early and effective treatment is recommended for the successful management of AMPE ${ }^{5-7}$. The European Society of Cardiology (ESC) has divided the prognostic risk into three groups: High-risk, medium-risk and low-risk ${ }^{5}$. Patients presenting with right ventricular (RV) dysfunction and hemodynamic instability are in the high-risk group ${ }^{5}$. The mechanisms responsible for myocardial damage in patients with PE in the high-risk group are unclear ${ }^{5,6}$; however, when patients present with concomitant heart failure, they typically have elevated cardiac troponin I (CTnl) levels ${ }^{7,8}$. Elevated CTnl expression is negatively correlated with patient prognosis ${ }^{9}$. Elucidating the mechanisms and factors that precipitate myocardial damage in patients with PE may be useful for predicting and improving patient prognoses.

AMPE damages the vascular endothelium, induces platelet activation and increases the plasma concentration of thromboxane $A 2\left(\mathrm{TXA2}^{10}\right.$, which leads to prostaglandin 12 (PGI2) metabolic disorders and elevated plasma concentrations of endothelin-1 $(\mathrm{ET}-1)^{11,12}$. Elevated plasma TXA2, PGI2 and ET-1 concentrations may cause pulmonary thrombosis and hypertension, which may further aggravate pulmonary embolism and exacerbate the myocardial damage associated with pulmonary embolism ${ }^{10-12}$.

Inhaled nitric oxide (NO) has been reported as an effective treatment for AMPE due to its vasodilatory effects ${ }^{13,14}$, as well as its ability to reduce neutrophil chelation, promote lung endothelium integrity, improve lung ventilation/perfusion, increase the density of blood vessels and repair vascular endothelium to restore injured lung tissue $e^{15,16}$.

$\mathrm{NO}$ is an important signaling molecule in the cardiovascular system and is considered to be a cardioprotective mediator ${ }^{17}$. However, its cardioprotective role in AMPE requires further investigation.

In the present study, a rabbit model with acute MPE-induced myocardial damage (AMPE$M D$ ) was prepared to study the mechanism of myocardial damage in MPE and correlations between the plasma concentrations of CTnl, TXA2, PGI2 and ET-1, were examined. The curative effect of NO inhalation (NOI) AMPE$M D$ was assessed and the results revealed that NOI may be an effective treatment for AMPE.

\section{Methods}

The present study was performed in strict accordance with the recommendations of the Guide for the Care and Use of Laboratory Animals of the National Institutes of Health (Bethesda, MA, USA). The animal use protocol has been reviewed and approved by the Medical Ethics Committee of Hebei University (approval number 21041109).

A total of 30 white Japanese rabbits aged 6-9 months and weighing 2-2.5 kg were provided by the Laboratory Animal Center, Medical Department of Hebei University (Baoding, China). Animals were housed at a temperature of $16-28^{\circ} \mathrm{C}$ with a humidity of 40 $70 \%$ and a $14 / 10 \mathrm{~h} \mathrm{light/dark}$ cycle. All rabbits received two meals at 09:00-09:30 and 16:0016:30 consisting of, 50-75 g pre-meal and $a d$ libitum access to water. The ratio of male: female rabbits was 1:1. Rabbits were randomly divided into the experimental ( $E X P, n=10), N O I$ $(n=10)$ and control (CON, $n=10)$ groups. The EXP group underwent modeling alone without any anti-coagulant therapy, rabbits in the NOI group received NOI $2 \mathrm{~h}$ post-modeling and the CON group received intravenous physiological 
saline instead of emboli. Rabbits in the EXP and NOI groups were treated with first-line therapy (dopamine; Changzhou Yabang Pharmaceutical Co., Ltd, Changzhou, China; $10-20 \mu \mathrm{g} / \mathrm{kg} / \mathrm{min}$, intravenous as required following modeling) to stabilize vital signs. The EXP and NOI groups did not receive the subcutaneous injection of low molecular heparin calcium as anti-coagulant therapy. Mechanical ventilation was the same in all groups.

\section{Preparation of rabbit AMPE-MD model}

Following disinfection and injection of $20 \%$ urethane (Shanghai Debang Chemical Co., Ltd., Shanghai, China) via the ear vein $(1.0 \mathrm{~g} /$ $\mathrm{kg}$ ), then urethane $0.5 \mathrm{~g} / \mathrm{kg}$ was maintained in the whole experiment. The rabbits underwent tracheal cannulation to facilitate spontaneous breathing and ensure protection of the airway.

A $2 \mathrm{~cm}$ conventional transverse incision was made on the anterior chest. The right jugular vein and left external carotid artery were separated and the left femoral vein was exposed and clearly separated. A pulmonary artery catheter (made in house) was inserted simultaneously and a microvascular catheter (5Fr TI; Tyler \& Company, Atlanta, GA, USA) was inserted through the right jugular vein and left carotid artery. Mechanical ventilation was applied. Pulmonary artery catheterization was monitored throughout the process using an oscilloscope to determine the positions of catheter tips based on pressure waveforms detected by the oscilloscope and the inserted length of the pulmonary catheters. Throughout the experiment, the pulmonary artery catheters were fed with physiological saline $(0.3 \mathrm{ml} / \mathrm{min})$ continuously using one micro-peristaltic pump (Cole-Parmer Instrument Company, LLC, St. Neots, UK). The two catheters were connected to one multi-channel physiological parameter analyzer (MP150; BIOPAC, Goleta, CA, USA) for synchronous measurement of the mean arterial pressure and mean pulmonary arterial pressure
(mPAP). The left femoral vein was punctured and implanted with one microcatheter for in-time rehydration and blood sampling. Rabbits were repeatedly injected (once every $3 \mathrm{~min}$ ) with $0.5 \mathrm{ml}$ of prepared emboli into the right jugular vein via the pulmonary artery catheter using a $5 \mathrm{ml}$ syringe, followed by 2 $\mathrm{ml}$ of physiological saline, until the MPAP was reduced to $40 \%$ of the baseline measurement and hypotension was maintained at 55-60 $\mathrm{mmHg}$, the heart beats were between 120160 per min, the respiratory rate was more than 40 per $\min$ for $\sim 40 \mathrm{~min}$, at which point the AMPE-MD model was considered to be successful. Rabbits in the CON group received saline only. During this process a ventilator for short-term mechanical ventilation was used if the rabbit developed serious breathing difficulties. Rabbits in the NOI group received NOI following modeling (Inhaled concentration was $10-20$ ppm immediately following surgery; administered for $2 \mathrm{~h}$ ). Rabbits in all groups received mechanical ventilation at the same time following tracheotomy using a SERVO-i infant ventilator (Rontgenvagen 2, SE-171 54; Maquet Critical Care AB, Solna, Sweden) with $50 \%$ oxygen.

\section{Perfusion and observation of results}

Following modeling, rabbits were perfused with acrylonitrile butadiene styrene (ABS) perfusion agent (made of byacrylonitrile butadiene and styrene) and subjected to BIOPAC pressure monitoring $(18-25 \mathrm{mmHg}$; BIOPAC).

After induction of adequate anesthesia by $20 \%$ urethane (Shanghai Debang Chemical Co., Ltd., Shanghai, China) via the ear vein (1000 mg/kg). Full experiment anaesthesia was maintained by injecting additional doses of the drug $(500 \mathrm{mg} / \mathrm{kg}$ i.v, ). Rabbits were fixed on the operating table. Fur was removed from the chest and abdomen and a $6 \mathrm{~cm}$ incision was made along the linea alba to expose the 
abdominal organs. The abdominal aorta was exposed, freed and threaded for further use. Prepared heparin (GSK, Middlesex, UK) saline was connected to the infusion device and, using a cutting needle, abdominal aortic cannulation, knotting, and fixation were performed, cutting the accompanying inferior vena cava and using the infusion device to rinse the blood vessels. Heparin rinsing was discontinued when the washed lung lobe appeared pale. A $50 \mathrm{ml}$ syringe was used to inject $50 \mathrm{ml}$ of acetone until the lung tissue exhibited a color change (the embolism became white, whilst the remainder became red) and the inferior vena cava emitted a strong odor. The thoracic cavity and manubrium were opened to ligate the superior vena cava. A catheter was inserted into the inferior vena cava above the diaphragm and connected to a BIOPAC multi-function pressure detector. The prepared ABS perfusion fluid was injected at a pressure of $18-25 \mathrm{mmHg}$ until the pressure continued to rise and could not be reduced, at which point the injection was discontinued. The lung was removed for Micro computed tomography scanning (PerkinElmer, Inc., Waltham, MA, USA.). Lung and heart tissue specimens were fixed in $10 \%$ formaldehyde at room temperature and for $48 \mathrm{~h}$, dehydrated and embedded in paraffin. Tissues were sectioned into 4-6 $\mu \mathrm{m}$ and Hematoxylin-Eosin stained (Hematoxylin stained for $5 \mathrm{~min}$ and Eosin for 1-2 $\mathrm{min}$ ) at room temperature for microscopic observation using an optical microscope (magnification, $\mathrm{x} 200$ ) and pathological assessment.

\section{Method of NOI}

Following successful modeling, mechanical ventilation was initiated when mPAP reached $40 \%$ and the rabbit exhibited breathlessness or breathing difficulties for $4 \mathrm{~h}$. NO was composed of $800 \times 10^{-6} \mathrm{~g} / \mathrm{l}$ decompressed NO, nitrogen (N2), oxygen (O2) and air. When the concentration of NOI reached $10-20 \times 10^{-6} \mathrm{~g} / \mathrm{L}$, a ventilator pipe was connected to the NOI pipe for invasive mechanical ventilation (pressure, $20 \mathrm{~cm}$ water column; respiratory rate, 28 bpm; oxygen concentration with mechanical ventilation, $50 \%)$. The concentrations of NO and nitrogen dioxide $\left(\mathrm{NO}_{2}\right)$ were continuously monitored using a nitrogen oxide analyzer (Thermo Fisher Scientific, Inc., Waltham, MA, USA) and the level of methemoglobin was also monitored throughout and did not exceed $0.3 \mathrm{~g} / \mathrm{L}$. Blood samples were obtained from Jugular vein at different time points $(0,2,4,8,12,16,20$ and $24 \mathrm{~h}$ ) and the mean arterial pressure and mPAP were monitored.

Plasma concentrations of CTnI, TXB2, PGI2 and ET-1

The plasma concentration of CTnl was determined using a microparticle chemiluminescence method with an automatic immunoassay analyzer (Thermo Fisher Scientific, Inc.). The immunoassay kit was provided by Beckman Coulter, Inc. (cat. no. ab47003; Brea, CA, USA). TXA2 and PGI2 are metabolic products of arachidonic acid, which are unstable and are further metabolized into TXB2 and 6-keto prostaglandin F1 $\alpha$ $(6-K-P G F 1 \alpha)^{18}$ The plasma concentrations of TXB2 (cat. no. 519031-96) and 6-K-PGF1 $\alpha$ (cat. no. C507164) were determined using kits (Beijing North Institute of Biological Technology, Beijing, China) while ET-1 was detected using a radioimmunoassay kit obtained from the Institute of Radioimmunology, Science and Technology Development Center, Beijing PLA General Hospital. All procedures were performed according to the manufacturer's protocol.

\section{Statistical analysis}

Data are expressed as the mean \pm standard deviation and were analyzed using 
SPSS statistical software 19.0 (IBM Corp., Armonk, NY, USA). One-way analysis of variance (ANOVA) followed by the Dunnett's multiple comparisons test and paired Student's twotailed $t$ tests were used. Correlation analyses were performed using Pearson correlation analysis. $\mathrm{P}<0.05$ was considered to indicate a statistically significant difference.

\section{- Results}

\section{Pathological results}

The embolized lungs were harvested and visualized using computed tomography
(Figure $1 \mathrm{~A}$ and $\mathrm{B}$ ). Following perfusion, the embolized lung lobes appeared pale (Figure 1C) and emboli were observed in the pulmonary arteries (Figure 1D). The right ventricular wall was thinner in rabbits from the EXP group compared with those in the NOI group (Figure $1 \mathrm{E}$ and $\mathrm{F})$. A greater degree of myocardial necrosis was observed in tissues from the EXP group compared with the NOI group (Figure $1 \mathrm{G}$ and $\mathrm{H}$ ). Cells exhibited disappearing striated cytoplasm, forming tone bands, with nucleation or disintegration. Inflammatory cell infiltration was also observed, the cell structure is blurred, nuclear debris was found and inflammatory cells are invaded.
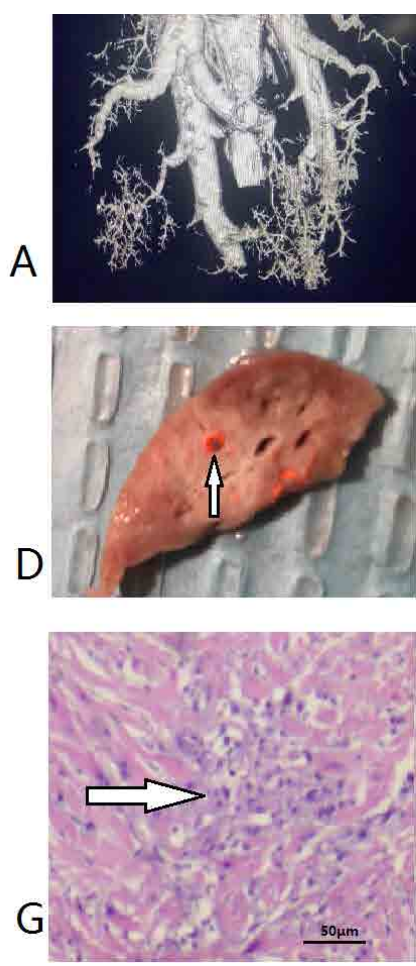
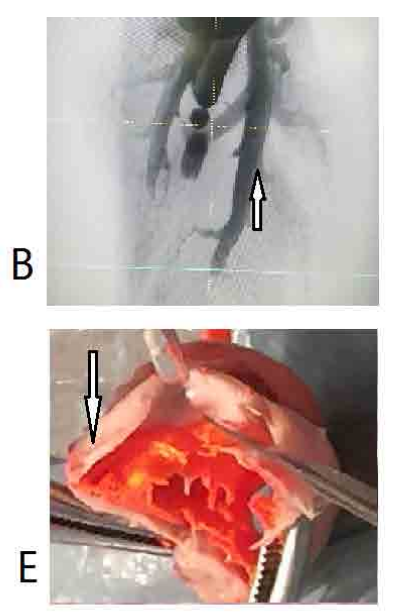

C
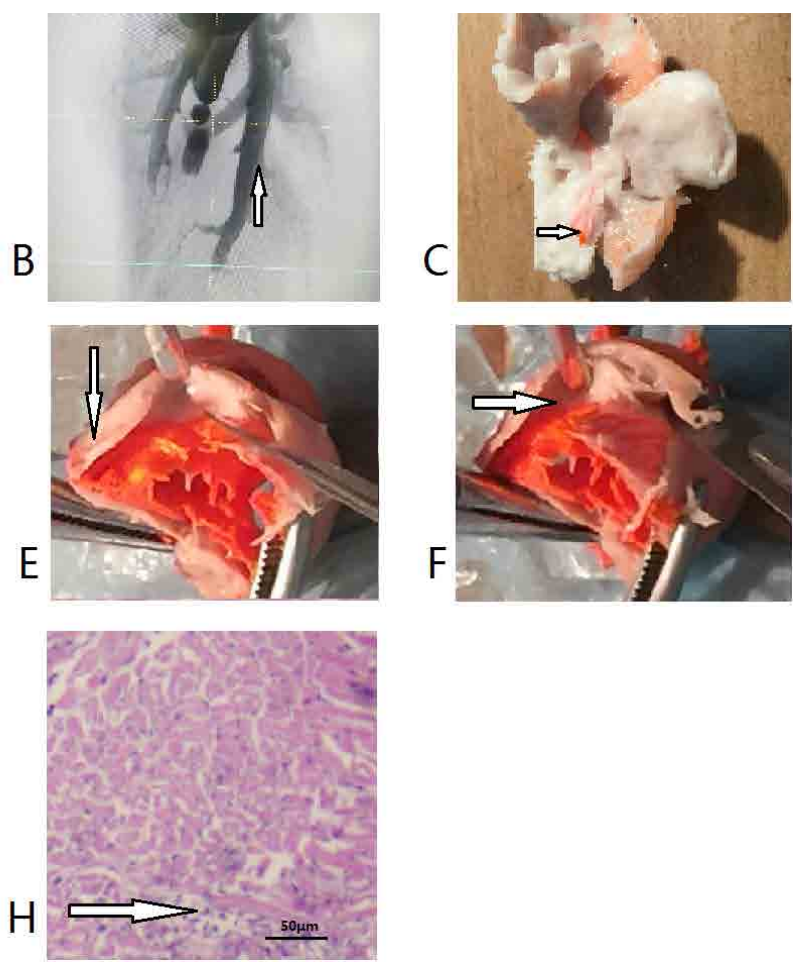

Figure 1 - Images and pathology of pulmonary embolism. (A) Computed tomography (B) X-ray angiography of pulmonary embolisms. (C) Arrow indicating embolized arteries in whole lungs following modeling. (D) Arrow indicating an embolized pulmonary lobe of a cross-sectioned lung following modeling. Arrow indicates the embolus. The right ventricle of a model rabbit (E) following and (F) prior to NOI. Pathological changes (G) prior to and $\mathbf{( H )}$ following NOI. A greater amount of myocardial necrosis is evident prior to NOI. Magnification, $\mathrm{x} 40$. NOI, nitric oxide inhalation. 


\section{Changes in plasma CTnI concentration}

The plasma CTnl concentration in the EXP group began to increase at $2 \mathrm{~h}$ following modeling and significantly increased at $4 \mathrm{~h}$, reaching a peak at $16 \mathrm{~h}$. CTnl concentrations were significantly higher in the EXP group compared with the CON group at 4, 8, 12, 16, 20 and $24 \mathrm{~h}$ (Table 1, Figure 2). Furthermore, $\mathrm{CTnl}$ concentrations were significantly higher in the EXP group compared with the NOI group at 4, 8, 12 and $16 \mathrm{~h}$ (Figure 2A).

\section{Changes in plasma TXB2 concentration}

The plasma TXB2 concentration in the EXP group began to increase $2 \mathrm{~h}$ following modeling and peaked at $12 \mathrm{~h}$. The TBX2 concentration in the EXP group was significantly higher compared with the CON and NOI groups at 4, 8, 12 and $16 \mathrm{~h}$ (Table 1, Figure 2B).

Changes in plasma 6-K-PGF1 $\alpha$ concentration

The plasma 6-K-PGF1 $\alpha$ concentration in the EXP group began to increase $2 \mathrm{~h}$ following modeling and peaked at $16 \mathrm{~h}$ (Figure $2 \mathrm{C}$ ). The plasma 6-K-PGF1 $\alpha$ was significantly lower in the CON group at $8,12,16,20$ and $24 \mathrm{~h}$ and in the NOI group at 8,12 and $16 \mathrm{~h}$ (Figure 2C) compared with the EXP group.

\section{Changes in plasma ET-1 concentration}

The plasma ET-1 concentration in EXP group began to increase $2 \mathrm{~h}$ following modeling and reached its peak concentration at $8 \mathrm{~h}$. ET-1 levels were significantly lower in the CON group at 2, 4, 8 and $12 \mathrm{~h}$ and in the NOI group at 2, 4 and $8 \mathrm{~h}$ (Table 1, Figure 2D) following modeling compared with the EXP group. The increase in plasma ET-1 concentration occurred earlier compared with the increase in plasma TXA2 concentration, whereas the increase in plasma 6-K-PGF concentration immediately followed that of TXA2. Changes in plasma ET1, TXA2 and 6-K-PGF concentrations prior to and following the administration of NOI were similar.

Table 1 - CON Group plasma ET-1, 6-K-PGF, TXB2, CTnl concentrations in test animals $(\bar{x} \pm s, \mathrm{n}=10)$.

\begin{tabular}{ccccccccc}
\hline Items & $\mathbf{O h}$ & $\mathbf{2 h}$ & $\mathbf{4 h}$ & $\mathbf{8 h}$ & $\mathbf{1 2 h}$ & $\mathbf{1 6 h}$ & $\mathbf{2 0 h}$ & $\mathbf{2 4 h}$ \\
\hline $\begin{array}{c}\mathrm{ET}-1 \\
(\mathrm{pg} / \mathrm{ml})\end{array}$ & $46.5 \pm 4.9$ & $46.7 \pm 5.1$ & $45.9 \pm 5.3$ & $46.2 \pm 5.9$ & $45.9 \pm 5.1$ & $46.2 \pm 5.4$ & $45.9 \pm 5.4$ & $46.2 \pm 5.6$ \\
$\begin{array}{c}6-\mathrm{K}-\mathrm{PGF} \\
(\mathrm{pg} / \mathrm{ml})\end{array}$ & $82.5 \pm 6.9$ & $84.7 \pm 8.4$ & $85.6 \pm 9.3$ & $81.6 \pm 6.9$ & $83.4 \pm 7.8$ & $84.6 \pm 6.2$ & $84.2 \pm 7.9$ & $84.7 \pm 6.6$ \\
$\begin{array}{c}\mathrm{TXB2} \\
(\mathrm{ng} / \mathrm{ml})\end{array}$ & $74.8 \pm 15.4$ & $72.5 \pm 18.1$ & $71.3 \pm 16.8$ & $70.5 \pm 16.1$ & $71.8 \pm 16.2$ & $73.3 \pm 15.9$ & $72.9 \pm 15.3$ & $72.7 \pm 16.8$ \\
$\begin{array}{c}\mathrm{CTnl} \\
(\mathrm{ug} / \mathrm{ml})\end{array}$ & $0.06 \pm 0.01$ & $0.07 \pm 0.01$ & $0.07 \pm 0.01$ & $0.08 \pm 0.01$ & $0.06 \pm 0.01$ & $0.06 \pm 0.01$ & $0.07 \pm 0.01$ & $0.08 \pm 0.01$ \\
\hline
\end{tabular}


A
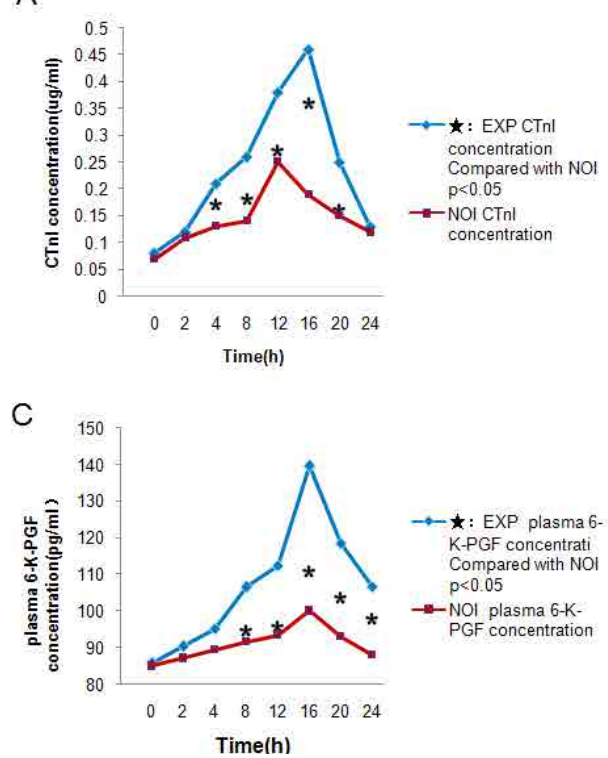

B

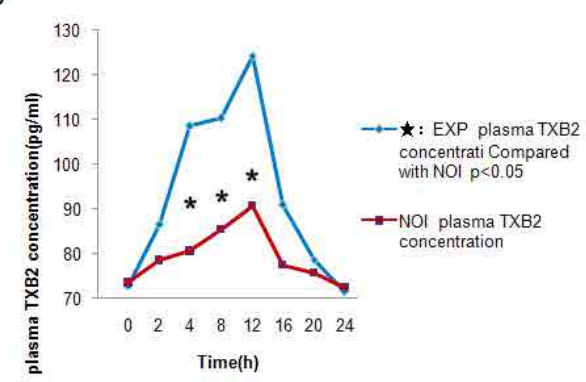

D

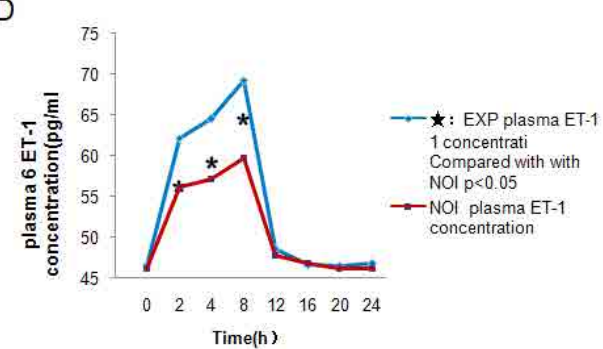

Figure 2 - Serum levels of CTnI, TXA2, 6-K-PGF1 $\alpha$ and ET-1 in the EXP and NOI groups. ${ }^{*} \mathrm{P}<0.05$ vs. EXP. CTnl, cardiac troponin I; TXA2, thromboxane A2; 6-K-PGF1 $\alpha$, 6-keto prostaglandin F1 $\alpha$; ET-1, endothelin-1; EXP, experimental; NOI, nitric oxide inhalation.

\section{Correlation between CTnl and peak plasma concentrations of TXB2, 6-K-PGF1 $\alpha$, and ET-1}

A marked positive association was observed in EXP group between CTnl and TXB2 (Figure 3A), CTnl and PGI2 (Figure 3B), and CTnI and ET-1 (Figure 3C).
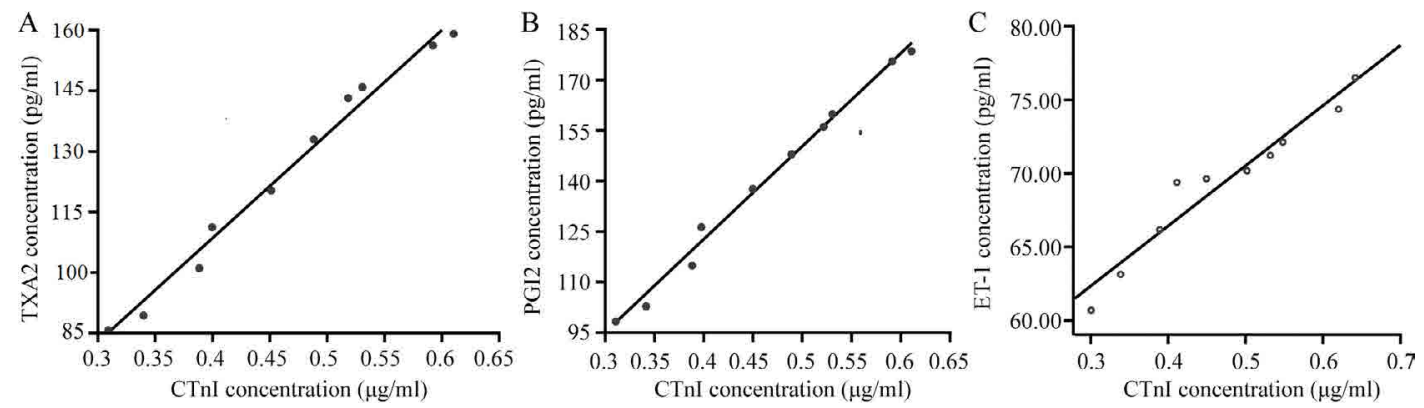

Figure 3 - Correlation between CTnl peak concentration and (A) TXA2, (B) PGI2; and (C) ET-1. CTnl, cardiac troponin I; TXA2, thromboxane A2; PG12, prostaglandin 12; ET-1, endothelin-1

\section{Discussion}

The results of the present study demonstrate that plasma TXA2 concentration is increased in rabbits with AMPE and its peak concentration in the plasma is significantly positive correlated with CTnl. CTnl is a marker for myocardial damage ${ }^{9}$, indicating that TXA2 serves an important role in AMPE-MD. A possible mechanism for this is that movement of emboli through the pulmonary vessels causes vascular endothelial damage, leading to activation of platelets and subsequent adsorption on the surface of the emboli. These activated platelets undergo degranulation to release TXA2 ${ }^{10,19,20}$. AMPE leads to ischemia, hypoxia and acidosis in rabbits; $\mathrm{H}^{+}$promotes platelet degranulation to release TXA2, which further increases the formation and enlargement of emboli, thereby forming a vicious cycle ${ }^{21-23}$ and 
aggravating right heart failure. Furthermore, AMPE-induced ischemia and hypoxia damages the coronary endothelium and these local and systemic factors act synergistically to enhance platelet activation and cause coronary artery thrombosis, myocardial ischemia and hypoxia, all of which result in myocardial damage and necrosis ${ }^{22,23}$.

The present study demonstrated that plasma PGI2 concentration increases in rabbits with AMPE and peak PGI2 concentration is significantly positively correlated with CTnI. $P G I 2$ is primarily associated with the inhibition and prevention of platelet aggregation ${ }^{24}$, relaxation of vascular smooth muscles and vasodilatation, particularly in capillaries and resistance vessels ${ }^{25}$. Plasma PGI2 concentrations were significantly increased in rabbits with $A M P E$, which may be due to the fact that blood vessels are mechanically blocked by emboli following modeling, which increases vascular pressure. The emboli and high blood flow subsequently provide a shear force that damages the vascular endothelium to precipitate the release of $P G I 2$, which is significantly delayed compared to TXA2. The increase in plasma PGI2 concentration may therefore act as a protective response to counteract/neutralize the increase of TXA2, which is most likely a reactionary increase. When TXA2 increases, PGI2 levels increase as a compensatory mechanism to maintain a balance between TXA2 and PGI 2 levels.

The results of the present study demonstrate that plasma ET-1 concentration is upregulated in a AMPE model. This may be because emboli cause mechanical injury to the pulmonary vascular endothelium, leading to pulmonary ischemia, hypoxia or acidosis, followed by sustained structural damage to and dysfunction of the pulmonary vascular endothelium, as well as an increased release of ET-1 ${ }^{11}$. The damaged pulmonary vasculature is subsequently unable to eliminate ET-1 in the blood $^{12}$, which leads to a significantly elevated plasma ET-1 concentration.

Elevated plasma ET-1 concentration causes extensive contraction of pulmonary vessels, increased resistance in the pulmonary circulation and secondary pulmonary hypertension ${ }^{26}$. Furthermore, mechanical blockage of the pulmonary vasculature secondary to emboli may also significantly increase pulmonary artery pressure ${ }^{27}$. The initial effect of AMPE on pulmonary circulation is an increase in pulmonary resistance ${ }^{28}$. In the early stages of AMPE there is an increase in right ventricular post load, causing an increase in the energy consumed by the right ventricle per beat, increased right ventricular end-stage systolic pressure and enlargement of the right ventricle ${ }^{29}$.

This in turn significantly reduces right ventricular output. Additionally, ischemia and hypoxia damage the coronary endothelium, inducing increased production/release of ET-1 in the coronary arteries ${ }^{22}$. These factors act synergistically to cause severe coronary artery vasoconstriction $^{22}$. Due to these effects, the coronary blood supply dramatically decreases, causing myocardial ischemia and injury and/or myocardial necrosis. The results of the present study demonstrated that, in rabbits with AMPE, the increase in plasma ET-1 occurred earlier compared with the other substances ( $2 \mathrm{~h}$ following modeling) and peaked at $8 \mathrm{~h}$. Furthermore, this increase was positively correlated with plasma CTnl concentrations, indicating that the increased plasma ET-1 concentration serves a key role in inducing myocardial damage in rabbits with AMPE. This is consistent with the fact that the initial impact of AMPE on the pulmonary circulation is an increase in pulmonary resistance, affecting the stability of the circulatory system and causing early myocardial damage ${ }^{22}$. It was also observed that the increase in plasma ET-1 concentration occurs earlier than the increase in TXA2 and that the consequent increase of ET-1 and TXA2 is associated with 
the AMPE-regulated synthesis of TXA2. The increase in plasma $P G I 2$ is correlated with increased TXA2 concentration and serves as a compensatory mechanism to maintain the balance between TXA2 and PGI2 levels. Increased concentrations of ET-1 and TXA2, as well as the balance maintained between TXA2 and PGI2, may serve a key role in the increased resistance in pulmonary circulation, pulmonary hypertension, and myocardial damage.

This present study demonstrated that $\mathrm{NOI}$ is able to significantly reduce plasma concentrations of TXA2, PGI2, ET-1 and CTnI in rabbits with AMPE. NOI may produce this beneficial effect inhibiting inhibiting the activity of protease $\mathrm{C}^{30}$, which inhibits the deregulation of activated platelets as well as reducing platelet aggregation and TXA2 production. NOI treatment may therefore reduce plasma TXA2 concentration, inhibit platelet aggregation and block multiple pathogenic steps in the development of AMPE. $\mathrm{NOI}$ also reduced plasma PGI2 concentrations, which may be responsible for maintaining the TXA2/PGI2 balance. This suggests that the TXA2/PGI2 ratio balance is more important that independent effect of PGI2 in pulmonary thromboembolism. NOI is able to reduce the plasma concentrations of ET-1 by reducing pulmonary arterial pressure and regulating the balance between TXA and TXA2/PGI2. The reduction in plasma ET-1 concentration in turn affects the levels of these other factors. NOI appears to effectively protect against myocardial damage and alleviate myocardial damage in PE via reducing plasma TXA2, PGI2 and ET-1.

AMPE treatment with NO remains a controversial topic and has not been widely used in the clinic. Kline et al. ${ }^{31}$ reported that the NOI combined with oxygen alleviated right ventricular dysfunction in patients with pulmonary thromboembolism and decreased the level of high-sensitivity troponin, which is not consistent with the results of the present study. Diasjunior et al. ${ }^{32}$ also demonstrated that intravenous administration of NO was not able to reduce pulmonary hypertension caused by AMPE. The use of high dose intravenous NO does not exhibit a selective effect on the pulmonary circulation, which can lead to severe systemic hypotension. When NO and vasodilatation are combined, the use of intravenous NO may not result in additional pulmonary vasodilation ${ }^{31}$. It has been reported that NOI as an auxiliary or palliative treatment for acute pulmonary thrombosis was able to reduce respiratory frequency and respiratory difficulties and improve the function of the right ventricle, as well as the average shock index ${ }^{13}$.

\section{- Conclusions}

Plasma concentrations of TXA2, PGI2 and ET-1 are significantly increased in MPE and are positively correlated with myocardial damage. NOI is able to reduce plasma concentrations of TXA2, PGI2 and ET1 , maintaining the balance between TXA2 and PGI2 to protect against myocardial damage. These results provide a theoretical basis for the treatment of pulmonary thromboembolism with clinical NOI.

\section{- References}

1. Darze ES, Casqueiro JB, Ciuffo LA, Santos JM, Magalhães IR, Latado AL. Pulmonary embolism mortality in Brazil from 1989 to 2010: gender and regional disparities. Arq Bras Cardiol. 2016;106(1):4-12. doi: 10.5935/abc.20160001.

2. Yavuz S, Toktas F, Goncu T, Eris C, Gucu A, Ay D, Erdolu B, Tenekecioglu E, Karaagac K, Vural $H$, Ozyazicioglu A. Surgical embolectomy for acute massive pulmonary embolism. Int J Clin Exp Med. 2014;7(12):5362-75. PMID: 25664045.

3. Dalen JE, Alpert JS. Natural history of pulmonary embolism. Prog Cardiovasc Dis. 1975;17(4):259-70. PMID: 1089991. 
4. Soloff LA, Rodman T. Acute pulmonary embolism. II. Clinical. Am Heart J. 1967;74(6):829-47. PMID: 6073360.

5. Jones $A E$, Watts JA, Debelak JP, Thornton LR, Younger JG, Kline JA. Inhibition of prostaglandin synthesis during polystyrene microsphere-induced pulmonary embolism in the rat. Am J Physiol Lung Cell Mol Physiol. 2003;284(6):L1072-81. doi: 10.1152/ ajplung.00283.2002.

6. Cho JH, Kutti Sridharan G, Kim SH, Kaw $R$, Abburi $T$, Irfan A, Kocheril AG. Right ventricular dysfunction as an echocardiographic prognostic factor in hemodynamically stable patients with acute pulmonary embolism: a meta-analysis. BMC Cardiovasc Disord. 2014;14:64. doi: 10.1186/1471-2261-14-64.

7. Meyer T, Binder L, Hruska $N$, Luthe $H$, Buchwald AB. Cardiac troponin I elevation in acute pulmonary embolism is associated with right ventricular dysfunction. J Am Coll Cardiol. 2000;36(5):1632-6. PMID: 11079669.

8. French Intensive Care Society, International congress - Réanimation 2016. Ann Intensive Care. 2016;6(Suppl 1):50. doi: 10.1186/ s13613-016-0114-z.

9. Kreit JW. The impact of right ventricular dysfunction on the prognosis and therapy of normotensive patients with pulmonary embolism. Chest. 2004;125(4):1539-45. PMID: 15078772.

10.Zhou B, Sun G, Mei F, Xu H. The effects of low-molecular-weight heparin on lung and pulmonary artery injuries in acute pulmonary embolism rat model via platelet-derived growth factor- $\beta$. Saudi Pharm J. 2017;25(4):564-9. doi: 10.1016/j. jsps.2017.04.024.

11.Krebs J, Ferguson SJ, Nuss K, Leskosek B, Hoerstrup SP, Goss BG, Shaw S, Aebli N. Plasma levels of endothelin-1 after a pulmonary embolism of bone marrow fat. Acta Anaesthesiol Scand. 2007;51(8):110714. doi: 10.1111/j.1399-6576.2007.01369.x.

12.Werner F, Kojonazarov B, Gaßner B, Abeßer M, Schuh K, Völker K, Baba HA, Dahal BK, Schermuly RT, Kuhn M. Endothelial actions of atrial natriuretic peptide prevent pulmonary hypertension in mice. Basic Res Cardiol. 2016;111(2):22. doi: 10.1007/ s00395-016-0541-x.

13.Kline JA, Hernandez J, Garrett JS, Jones
AE. Pilot study of a protocol to administer inhaled nitric oxide to treat severe acute submassive pulmonary embolism. Emerg Med J. 2014;31(6):459-62. doi: 10.1136/ emermed-2013-202426.

14.Trummer G, Berchtold-Herz M, Martin J, Beyersdorf F. Successful treatment of pulmonary hypertension with inhaled nitric oxide after pulmonary embolectomy. Ann Thorac Surg. 2002;73(4):1299-301. PMID: 11996278.

15. Waldow T, Witt $W$, Janke $A$, Ulmer A, Buzin A, Matschke K. Cell-cell junctions and vascular endothelial growth factor in rat lung as affected by ischemia/reperfusion and preconditioning with inhaled nitric oxide. J Surg Res. 2009;157(1):30-42. doi: 10.1016/j.jss.2008.07.042.

16.Qi Y, Qian L, Sun B, Liu L, Wu P, Sun L. Inhaled NO Contributes to lung repair in piglets with acute respiratory distress syndrome via increasing circulating endothelial progenitor cells. PLoS One. 2012;7(3):e33859. doi: 10.1371/journal.pone.0033859.

17.Strijdom H, Chamane N, Lochner A. Nitric oxide in the cardiovascular system: a simple molecule with complex actions. Cardiovasc J Afr. 2009;20(5):303-10. PMID: 19907806.

18. Preeyasombat C, Treepongkaruna S, Sriphrapradang A, Choubtum L. The role of prostacyclin (PGI2) and thromboxane A2 (TXA2) in pathogenesis of dengue hemorrhagic fever (DHF). J Med Assoc Thai. 1999;82 Suppl 1:S16-21. PMID: 10730512.

19.Mizurini DM, Aslan JS, Gomes T, Ma D, Francischetti IM, Monteiro RQ. Salivary thromboxane A2-binding proteins from triatomine vectors of chagas disease inhibit platelet-mediated Neutrophil Extracellular Traps (NETs) formation and arterial thrombosis. PLoS Negl Trop Dis. 2015;9(6):e0003869. doi: 10.1371/journal. pntd.0003869.

20.Kim JH, Lee J, Kang S, Moon H, Chung KH, Kim KR. Antiplatelet and antithrombotic effects of the extract of linderaobtusiloba leaves. Biomol Ther (Seoul). 2016;24(6):65964. doi: 10.4062/biomolther.2016.021.

21.Kobayashi K, Horikami D, Omori K, Nakamura T, Yamazaki A, Maeda S, Murata T. Thromboxane $A 2$ exacerbates acute lung injury via promoting edema formation. Sci Rep. 2016;6:32109. doi: 10.1038/ srep32109. 
22.Lannan KL, Phipps RP, White RJ. Thrombosis, platelets, microparticles, and PAH: more than a clot. Drug Discov Today. 2014;19(8):12305. doi: 10.1016/j.drudis.2014.04.001.

23.Diamond SL. Systems analysis of thrombus formation. Circ Res. 2016;118(9):1348-62. doi: 10.1161/CIRCRESAHA.115.306824.

24. Mitchell JA, Ahmetaj-Shala B, Kirkby NS, Wright WR, Mackenzie LS, Reed DM, Mohamed N. Role of prostacyclin in pulmonary hypertension. Glob Cardiol Sci Pract. 2014;2014(4):382-93. doi: 10.5339/ gcsp.2014.53.

25.LeVarge BL. Prostanoid therapies in the management of pulmonary arterial hypertension. Ther Clin Risk Manag. 2015;11:535-47. doi: 10.2147/TCRM. S75122.

26.Benza RL, Gomberg-Maitland $M$, Demarco $T$, Frost $A E$, Torbicki A, Langleben D, Pulido $T$, Correa-Jaque $P$, Passineau MJ, Wiener HW, Tamari M, Hirota T, Kubo M, Tiwari HK. Endothelin-1 pathway polymorphisms and outcomes in pulmonary arterial hypertension. Am J Respir Crit Care Med. 2015;192(11):1345-54. doi: 10.1164/ rccm.201501-01960C.

27.Liu YY, Li XC, Duan Z, Yuan YD. Correlation between the embolism area and pulmonary arterial systolic pressure as an indicator of pulmonary arterial hypertension in patients with acute pulmonary thromboembolism. Eur Rev Med Pharmacol Sci. 2014;18(17):2551-5. PMID: 25268104.

28. Meyer G, Sors H, Charbonnier B, Kasper
W, Bassand JP, Kerr IH, Lesaffre E, Vanhove $P$, Verstraete $M$. Effects of intravenous urokinaseversusalteplaseontotalpulmonary resistance in acute massive pulmonary embolism: a European multicenter doubleblind trial. The European Cooperative Study Group for Pulmonary Embolism. J Am Coll Cardiol. 1992;19(2):239-45. PMID: 1732347. 29.Keller K, Beule J, Coldewey M, Geyer M, Balzer JO, Dippold W. The risk factor age in normotensive patients with pulmonary embolism: effectiveness of age in predicting submassive pulmonary embolism, cardiac injury, right ventricular dysfunction and elevated systolic pulmonary artery pressure in normotensive pulmonary embolism patients. Exp Gerontol. 2015;69:116-21. doi: 10.1016/j.exger.2015.05.007.

30. Rossaint R, Falke KJ, López F, Slama K, Pison $U$, Zapol WM. Inhaled nitric oxide for the adult respiratory distress syndrome. $\mathrm{N}$ Engl J Med. 1993;328(6):399-405. doi: 10.1056/ NEJM199302113280605.

31.Kline JA, Hall $C L$, Jones $A E$, Puskarich MA, Mastouri RA, Lahm T. Randomized trial of inhaled nitric oxide to treat acute pulmonary embolism: The iNOPE trial. Am Heart J. 2017;186:100-10. doi: 10.1016/j. ahj.2017.01.011.

32.Dias-Junior CA, Tanus-Santos JE. Hemodynamiceffects of sildenafilinteraction with a nitric oxide donor compound in a dog model of acute pulmonary embolism. Life Sci. 2006;79(5):469-74. doi: 10.1016/j. Ifs.2006.01.034.

\section{Correspondence:}

Zheng Li

Department of Respiratory Medicine,

Affiliated Hospital of Hebei University

212 Yuhua East Road Lianchi District

Baoding 071000, Hebei China

Phone: +86 3125981021

Fax: +863125981688

zhenlidoc@126.com

Received: Mar 06, 2018

Review: May 08, 2018

Accepted: June 09, 2018
Conflict of interest: none

Financial source: none
${ }^{1}$ Research performed at Animal Laboratory, Medical Research Center, Hebei University, Baoding, China. 